\title{
Study Subject Type
}

National Cancer Institute

\section{Source}

National Cancer Institute. Study Subject Type. NCI Thesaurus. Code C70835.

A target biological entity of the study. 